\title{
Sistema de evaluación de soluciones de rehabilitación energética para edificios bien de interés cultural (SESREBIC). Su aplicación a monasterios BIC
}

\section{Evaluation system for retrofitting solutions for energy saving in historic buildings (SESREBIC). Application in BIC monasteries}

$\underline{\text { M J. López-Zambrano }}^{(*)}$, J. Canivell ${ }^{(*)}$, C. Calama-González ${ }^{(* *)}$

\section{RESUMEN}

En el presente artículo, se resume la investigación llevada a cabo para diseñar un sistema que ayude a la toma de decisiones en relación a intervenciones de rehabilitación energética en edificios Bien de Interés Cultural. Los criterios relacionados con la viabilidad técnica y la mejora de la eficiencia energética, no son suficientes en el caso de intervenciones en edificios BIC, en los que deben analizarse los requisitos que fijan las Comisiones de Patrimonio, con el fin de aplicar en la actuación soluciones que mejoren su comportamiento energético sin alterar sus cualidades como BIC. Con este sistema se pretende dar una primera respuesta al RD 564/2017, que permite realizar las certificaciones de eficiencia energética en este tipo de edificios, si cumplen estos requisitos. La validez del sistema se ha comprobado evaluando soluciones para cuatro intervenciones en monasterios incluidos en el catálogo de BIC del Estado Español.

Palabras clave: Rehabilitación energética, Bien Interés Cultural, eficiencia energética, construcción patrimonial.

\section{ABSTRACT}

The present paper summarizes the research carried out in order to design a strategic tool to help in decision-making, with the aim of tackling the problem of retrofitting buildings officially protected, representative of Cultural Heritage Interest. In the case of energy retrofitting interventions in the Cultural Heritage, criteria based on technical viability and energy efficiency improvements should be analyzed comprehensively and in conjunction with qualities established by Heritage Committees, meeting the requirements of a solution that not only improves energy performance but also preserves cultural interests. Furthermore, this tool offers an initial response to the Royal Decree 564/2017, which establishes the certification of energy efficiency of these type of buildings. System validation has been conducted through an evaluation of four retrofitting interventions developed in monasteries, all of them considered Cultural Heritage Interest and included in the General Catalogue of the Spanish Historical Heritage.

Keywords: Energy retrofitting, heritage of cultural interest, energy efficiency, heritage building.

(*) National University of Ireland in Galway (Irlanda).

(*) Universidad de Sevilla (España).

Persona de contacto/Corresponding author: mlopez@eficienciaypatrimonio.com (M.J. López-Zambrano).

ORCID: https://orcid.org/oooo-0oo3-4558-3177 (M.J. López Zambrano); https://orcid.org/oooo-0001-7636-102X

(J. Canivell); https://orcid.org/oooo-0002-6511-2885 (C. Calama-González).

Cómo citar este artículo/Citation: López-Zambrano, M.J.; Canivell, J.; Calama-González, C. (2019). Sistema de evaluación de soluciones de rehabilitación energética para edificios bien de interés cultural (SESREBIC). Su aplicación a monasterios BIC. Informes de la Construcción, 71(555): e30o. https://doi.org/10.3989/ic.63532.

Copyright: (C) 2019 CSIC. Este es un artículo de acceso abierto distribuido bajo los términos de la licencia de uso y distribución Creative Commons Reconocimiento 4.0 Internacional (CC BY 4.0). 


\section{INTRODUCCIÓN}

El Real Decreto 235/2013 (1), por el que se aprueba el procedimiento básico para la certificación de la eficiencia energética de los edificios, significó un paso importante para fomentar la eficiencia energética en los edificios, incluidos los existentes. Pero dentro del ámbito de actuación se excluían los edificios y monumentos protegidos oficialmente en razón de su particular valor arquitectónico o histórico.

Según la base de datos de Bienes Inmuebles del Ministerio de Educación, Cultura y Deporte (2) existen 16.146 inmuebles declarados de Interés. A estos hay añadir los que a nivel local y provincial tienen algún grado de protección en el planeamiento.

Gran parte de ellos están en uso, como centros culturales, educativos, administrativos e incluso hoteleros, siendo importantes sus necesidades de mejora del rendimiento energético. Una intervención inapropiada podría ser irreversible, por lo que requiere un estudio meticuloso y específico, que combine el conocimiento de medidas adecuadas en materia de eficiencia energética y el cocimiento técnico, sensibilidad y respeto profundo por el Patrimonio para poder hallar las soluciones más idóneas sin afectar a los elementos protegidos.

La reciente aprobación del Real Decreto 564/2017 (3), que modifica el Real Decreto 235/2013, que aprueba el procedimiento básico para la certificación de la eficiencia energética de los edificios, ha abierto la puerta a la rehabilitación energética de los edificios protegidos, siempre que las actuaciones de mejora no alteren de manera inaceptable su carácter o aspecto.

Uno de los factores que presenta mayor dificultad para la aplicación de medidas para la mejora de la eficiencia energética en los edificios con protección, tiene su base en la ley de Patrimonio Histórico Español en vigor desde 1985 (4), que considera inalterables los aspectos y elementos singulares que han llevado a su declaración como BIC.

Otro problema, es que a este tipo de edificios es muy difícil aplicarles la normativa de eficiencia energética, ya que los procedimientos actuales y las guías que existen para realizar certificaciones energéticas (5), auditorías (6) o catálogos de soluciones de rehabilitación energética (7), recogen exclusivamente los datos necesarios para hacer posteriormente los cálculos o simulaciones en herramientas informáticas. Pero tanto, en estas herramientas informáticas (8) como en las guías y catálogos, no se tienen en cuenta los límites de actuación marcados por las catalogaciones de los edificios protegidos, de forma que podríamos obtener una mejora en la calificación energética, pero las soluciones propuestas podrían ser incompatibles con su carácter BIC.

Las soluciones de rehabilitación energética de un edificio BIC, deben considerar dos aspectos básicos. El primero es un análisis histórico y de su protección patrimonial, como paso previo que nos ayude a diseñar unas soluciones válidas y adecuadas para el edificio. Y, en segundo término, un estudio exhaustivo de las soluciones para la mejora que permita que la intervención pase el filtro de la Comisión Territorial de Patrimonio correspondiente que debe aprobar dichas medidas.

\section{ESTADO DE LA CUESTIÓN}

La entrada en vigor del RD 564/2017 ha abierto una puerta a la posibilidad de certificar energéticamente edificios con protección patrimonial. Hasta esta fecha, las intervenciones de rehabilitación energética sobre edificios BIC, se realizaban como intervenciones singulares, que se limitaban al análisis del edificio en cuestión, por lo que no eran válidas para su aplicación con carácter general. Es el caso, por ejemplo, del proyecto RENERPATH: Metodología de rehabilitación energética de edificios patrimoniales (9), en el que se ha desarrollado una metodología que posteriormente se aplicó en dos edificios protegidos, en Ciudad Rodrigo y en Portugal. Otros proyectos financiados por la Unión Europea, sobre eficiencia energética y Patrimonio Cultural, son EFFESUS (10) (enfocado a distritos urbanos, no a edificios) y 3ENCULT (11) (Centrado en la reducción de la demanda energética y la mejora de la habitabilidad en edificios históricos).

Se está investigando sobre la metodología de intervención, soluciones específicas, incorporación de herramientas informáticas para su seguimiento con GIS (12) o BIM (13)(14), incluso desarrollo de sensores específicos de medición de condiciones ambientales (15). Pero tratar los edificios protegidos como casos al margen de la normativa, deriva en que muchas de las herramientas y guías habituales se diseñen sin incluirlos. Lo ideal sería incluir los edificios catalogados en las metodologías y herramientas actuales, pero existen diferencias importantes en el tratamiento de edificios protegidos y no protegidos. En el caso de la elección de soluciones para la rehabilitación energética, sería necesario establecer un paso previo para rechazarlas o aceptarlas en función de unos requisitos patrimoniales preestablecidos, lo que facilitaría el proceso de elección soluciones y la integración de los BIC en los documentos o herramientas reconocidas por el Ministerio para certificar energéticamente.

Por esta razón esta investigación (16) propone generar unos criterios y un sistema de evaluación que puedan ser incorporados en las herramientas de calificación o evaluación existentes, para poder realizar la certificación energética de los edificios protegidos como BIC.

\section{HIPÓTESIS DE PARTIDA}

Se parte de la base de que los actuales sistemas de valoración de soluciones de rehabilitación energética tienen solo un fundamento económico y energético y no contemplan los elementos y sistemas del edificio que han servido de base para catalogarlo como BIC, por lo que es necesario el diseño de un sistema de valoración que también incluya los indicadores necesarios para respetar la protección patrimonial.

En las intervenciones en materia de rehabilitación energética de edificios BIC, existen dos tipos de limitaciones. Unas tienen su base en los marcos normativos que regulan las condiciones energéticas que deben cumplir, la otra es que deben respetar las condiciones de intervención que se regulan en su catalogación como BIC. Por lo tanto, es necesario dar una respuesta que facilite y promueva la generación de soluciones constructivas que, mejorando las condiciones energéticas del monumento, no afecten a su cualidad cultural y patrimonial. Con este sentido se proponen indicadores cualitativos y cuantitativos que permitan dar una respuesta adecuada a la rehabilitación desde la fase de proyecto. 
Como primera aproximación al problema, se ha considerado adecuado limitar el estudio a la evaluación de las soluciones de rehabilitación energética relacionadas con la envolvente, porque son los que afectan más directamente a los criterios de catalogación del BIC. No está incluido - por su envergadura- el estudio de las instalaciones, aunque se contempla su futura integración en el sistema de evaluación.

Para contextualizar la aplicación, como la tipología de BIC es muy amplia y diversa, la investigación se centra en la tipología constructiva del monasterio. Una de las razones para su elección es la existencia del listado incluido en el Plan Nacional de Abadías, monasterios y conventos (17) con más de 500 edificios, aunque ha sido necesario completarlo con una base de datos propia. Además, se trata de una tipología versátil, muy extendida y con una fisonomía y características constructivas muy reconocibles, pero que muestran -en general- una deficiencia energética, al no cumplir sus sistemas constructivos los estándares mínimos de confort térmico. Asimismo, actualmente muchos de estos edificios se están rehabilitando para albergar una gran variedad de usos que requieren una mejora de la eficiencia energética.

\section{METODOLOGÍA}

Para su desarrollo, la investigación se ha divido en varias fases:

A) Análisis y aproximación al problema desde el marco normativo y de experiencias anteriores.

B) Elaboración de base de datos de monasterios BIC y selección de casos de estudio para diseñar el sistema. Se han seleccionado tres monasterios BIC, con usos diferentes correspondientes a las tipologías más comunes. El monasterio de Santa María la Real (Aguilar de Campoo), acoge centros docentes, fundaciones y un museo; Santa María de la Vid (Burgos), que alberga una orden religiosa y hospedería y Santa María de Retuerta (Valladolid), que se ha transformado en hotel de lujo con elevados estándares de confort.

C) Diseño del sistema de indicadores y su valoración, tomando como base los sistemas constructivos de la envolvente térmica (suelos, muros, cubiertas y huecos), así como la categorización desde el punto de vista patrimonial del elemento constructivo que será rehabilitado.
D) Diseño del sistema de evaluación integrando primero indicadores de índole patrimonial y posteriormente los energéticos y constructivos.

E) Puesta en carga y refinamiento del sistema mediante la aplicación en los tres modelos.

F) Validación del sistema, realizando la evaluación de las soluciones de rehabilitación energética ejecutadas en el Monasterio de El Escorial, en los patios del Colegio Alfonso XII.

\section{PROPUESTA DEL SISTEMA}

El sistema consiste en una herramienta que permite emitir un juicio relevante sobre las cualidades que aporta una solución constructiva en la rehabilitación energética del BIC. Se trata de establecer una selección y ordenación cualitativa de soluciones, como asistencia en la toma de decisiones previa a la calificación o la simulación energética, excluyendo otras que no cumplen los requisitos de catalogación.

Por lo tanto, el sistema tiene dos funciones principales:

a) Validar o descartar una solución desde el punto de vista del cumplimiento de los requisitos de catalogación.

b) En el caso de cumplir la catalogación, proceder a la evaluación de la solución a nivel energético y constructivo.

\subsection{Categorías de catalogación}

Para poder determinar la viabilidad de una solución y sabiendo que existen diferentes situaciones que condicionan el elemento a intervenir, se establecen cinco categorías de catalogación del elemento (Tabla 1) que hemos denominado CASOS y condicionan la valoración de los indicadores.

\subsection{Indicadores del sistema}

Se utilizan tres tipos de indicadores. Indicadores relacionados con el cumplimiento de la calificación patrimonial del edificio (CAT) y que son básicos para su cumplimiento. Posteriormente se evalúan los indicadores relacionados con la rehabilitación desde su punto de vista constructivo (REH) y los relacionados con la rehabilitación energética (ENE). Para evaluar cada uno se establece una escala de valoración de tres

Tabla 1. Categorías o CASOS en que puede encontrarse el elemento constructivo sobre el que se va a intervenir.

\begin{tabular}{|l|l|l|}
\hline \multicolumn{1}{|c|}{ Categorías } & \multicolumn{1}{|c|}{ Descripción } & \multicolumn{1}{|c|}{ Palabras clave } \\
\hline $\begin{array}{l}\text { Elemento original con posibilidad de } \\
\text { restauración }\end{array}$ & $\begin{array}{l}\text { Elemento original protegido, buen } \\
\text { estado de conservación, sólo es posible la } \\
\text { Restauración }\end{array}$ & $\begin{array}{l}\text { Original Protegido } \\
\text { Sestauración }\end{array}$ \\
\hline $\begin{array}{l}\text { Elemento original sin posibilidad de } \\
\text { restauración, pero sí de rehabilitación }\end{array}$ & $\begin{array}{l}\text { Elemento original protegido al que hay que } \\
\text { integrarle una pieza nueva o sustituir por } \\
\text { otro nuevo al encontrase mal conservado }\end{array}$ & $\begin{array}{l}\text { Original Protegido } \\
\text { Rehabilitación }\end{array}$ \\
\hline Elemento original no protegido y/u oculto & $\begin{array}{l}\text { Elemento original no protegido o que } \\
\text { estando protegido se puede ocultar bajo } \\
\text { otra solución }\end{array}$ & $\begin{array}{l}\text { Original } \\
\text { No Protegido } \\
\text { Protegido } \\
\text { Oculto }\end{array}$ \\
\hline Elemento no original visto & $\begin{array}{l}\text { Elemento no original integrado en una } \\
\text { zona protegida }\end{array}$ & No Original \\
Elemento no original y/u oculto & $\begin{array}{l}\text { Elemento no original que puede } \\
\text { permanecer oculto }\end{array}$ & Visto \\
\hline
\end{tabular}

Indicadores del sistema. 
niveles, puntuando de 1 al 3, siendo normalmente 1 la valoración más desfavorable de indicador.

\subsubsection{Indicadores relacionados con el cumplimiento de la calificación patrimonial del edificio (CAT)}

Uno de los problemas planteados en el diseño del sistema es la elección de indicadores relacionados con el cumplimiento de los requisitos de la catalogación patrimonial, ya que cada Comunidad Autónoma y cada ayuntamiento tienen una norma propia. Por ello se parte del análisis de la Ley 16/1985 de Patrimonio Histórico Español (4) y de los criterios más comúnmente utilizados por legislaciones autonómicas y ayuntamientos. Este grupo de indicadores se denomina CAT, y condicionan la validación o el rechazo de la solución propuesta, ya que evalúan los requisitos establecidos en la catalogación patrimonial del edificio.

La determinación de los indicadores considera aspectos recogidos en las citadas leyes:

a) Mínima intervención. Respeto por la información histórica, los materiales tradicionales, los métodos de construcción y las características esenciales del bien, sin perjuicio de que pueda autorizarse el uso de elementos, técnicas y materiales actuales para la mejor conservación del mismo.

b) Se deben conservar las características volumétricas, estéticas, ornamentales y espaciales del inmueble, así como las aportaciones de distintas épocas. La eliminación de alguna de ellas deberá estar claramente documentada y convenientemente justificada en orden a la adecuada conservación de los bienes afectados.

c) Se evitarán los intentos de reconstrucción. Cuando la aportación de materiales sea indispensable para la estabilidad y el mantenimiento del inmueble, esta habrá de ser justificada, reconocible y sin discordancia estética o funcional con el resto del mismo. No podrán realizarse reconstrucciones que conduzcan a confusiones miméticas que falseen su autenticidad histórica, salvo cuando se utilicen partes originales de los mismos y pueda probarse su procedencia.

d) La reversibilidad de los procedimientos que se apliquen.

e) Las técnicas y materiales serán compatibles con los que conforman el bien y su entorno, o con los valores objeto de protección según el régimen aplicable. Las técnicas y materiales propuestos deberán ofrecer comportamientos y resultados suficientemente avalados por la experiencia o por la investigación.

f) Las adiciones que se autoricen deberán respetar la armonía del conjunto, distinguiéndose de las partes originales para evitar las falsificaciones históricas o artísticas.

g) En todos los casos, sólo se permite restauración o rehabilitación. En el caso de elementos originales existentes, se aboga por la restauración. Cuando estos elementos ya no existen o se encuentran en un mal estado, de modo que la restauración no sea posible, se decanta por la sustitución de los elementos dañados por otros nuevos, pero de similares características.

h) En cuanto a fachadas, prima el no alterar la composición original. No cambiar huecos y mantener el aspecto estético de los acabados.

i) En cubiertas, no alterarla altura y la morfología y que el elemento de cobertura original sea mantenido, al menos en aspecto. j) En estructuras, prevalecerá la restauración de elementos originales y la rehabilitación de elementos en mal estado, mayormente cuando son vistos. Si son elementos ocultos y no son originales, se puede intervenir con otros sistemas y materiales diferentes.

k) Respecto a carpinterías, se optará por restaurar los originales y si hubiera que sustituir, hacerlo con carpinterías de madera u otro material que imitará el aspecto del elemento original.

1) Para los suelos, mantener original y restituir con piezas de aspecto similar.

Para cumplir estos requisitos se han considerado seis indicadores CAT, cuyos criterios y valoraciones (en una escala de 1 a 3 puntos) detallamos:

a) COLOR (CAT_O1_co)

Es un indicador importante en la categoría de elementos protegidos originales (CASO1_OP_SR). En el caso de elementos originales protegidos sin posibilidad de restauración (CASO2_OP_RE), podrán realizarse restituciones siempre y cuando el acabado no se mimetice con el elemento original, por lo tanto, el color de la solución deberá ser similar. En el resto de los casos no sería necesario cumplir con este requisito para que la solución fuera válida. El sistema realiza la siguiente puntuación:

- Se considera del mismo color si el color y tono son iguales al del elemento existente o muy parecido y sólo se aprecia la diferencia desde muy corta distancia. (Valoración: 3).

- Similar, si es del mismo color, pero varia ligeramente su tonalidad, pudiéndose distinguir el elemento original del nuevo. (Valoración: 2).

- Nada parecido, cuando se trata de otro color. (Valoración: 1 ).

b) TEXTURA (CAT_O2_te)

Por su similitud al indicador anterior se aplican criterios similares:

- Textura idéntica y sólo se aprecia la diferencia al tacto. (Valoración: 3).

- Similar, si es similar al tacto, pero se percibe la diferencia. (Valoración: 2).

- Nada parecido, cuando se trata de otra textura. (Valoración: 1).

c) VOLUMEN (CAT_O3_vo)

Valora si el elemento sobresale de la alineación actual o si lo hace en altura. En general cuando en la catalogación se indica que debe conservarse el volumen, hace referencia a la imposibilidad de aumentar el volumen del edificio de forma sustancial. Sin embargo, se considera incluir una restricción mayor ya que muchas normas municipales imponen la conservación del volumen del elemento, pero dejan la opción de solicitar la modificación del mismo. Teniendo en cuenta que, en la mayoría de los casos, los monasterios son edificios aislados y exentos, solo se considera la restricción del volumen en las categorías CASO1_OP_SR y CASO2_OP_RE. Los criterios serían:

- El volumen es el mismo. Tanto en el caso de muros, suelos y huecos se mantiene el mismo espesor y/o altu- 
ra, salvo que la catalogación permita solicitar una ampliación del volumen. (Valoración: 3).

- Similar. Cuando en el caso de muros y cubiertas sobresale o eleva ligeramente su altura, pero sin alterar el aspecto compositivo del edifico; y en el caso de suelos su ligera alteración no afecta a huecos de paso y/o escaleras. (Valoración: 2).

- Nada parecido, si sobresale de manera sustancial de la alineación y/o de la rasante y en suelos supone alteraciones que obligan a modificar huecos de paso y/o escaleras. (Valoración: 1).

\section{d) APARIENCIA (CAT_04_ap)}

Hace referencia al diseño global. Como en los indicadores anteriores, en CASO1_OP_SR la apariencia deberá ser idéntica, en el CASO2_OP_RE podría tener un diseño similar, e igual en el CASO4_NO_V, si el elemento se encuentra integrado en una zona protegida, la solución debería tener aspecto similar al original. Se utilizan los siguientes criterios:

- El elemento es idéntico, es decir, la solución no varía la apariencia del elemento en diseño o composición, no podría distinguirse la solución del original. (Valoración: 3).

- Similar. La solución no varía la apariencia del elemento en diseño o composición, podría distinguirse la solución del original. (Valoración: 2).

- Nada parecido. (Valoración: 1).

No obstante, requiere puntualizar que para el CASO2 OP_RE, en las catalogaciones se establece que debe poder distinguirse el elemento original del nuevo. Por ello ambos se deben diferenciar mediante el color, la textura, el volumen y/o la apariencia. En nuestro sistema se establece una condición adicional por la que al menos uno de estos cuatro indicadores no puede alcanzar el nivel de idéntico (Valoración: 3). Así, para la categoría mencionada, sería posible distinguir entre original y nuevo.

e) COMPATIBILIDAD (CAT_O5_cm)

Evalúa que sea compatible desde el punto de vista físicoquímico con el elemento soporte. De forma que podamos garantizar la integridad y conservación del mismo. Siempre que un elemento sea original, aunque no estuviera protegido o pudiera permanecer oculto (CASO3_ONP PO), se debe garantizar la compatibilidad del elemento con la solución a adoptar. Cuando no son originales también sería recomendable, pero no tiene sentido considerarlo como requisito obligatorio. Los criterios y la valoración de este indicador serían:

- Compatible, cuando no hay ningún problema de interacción con el elemento de soporte. (Valoración: 3).

- Poco compatible, puede tener efectos perjudiciales a largo plazo. (Valoración: 2).

- Incompatible, cuando se desaconseja su utilización. (Valoración: 1).

\section{f) REVERSIBILIDAD (CAT_o6_re)}

Las recomendaciones de ICOMOS (18) establecen que la intervención debe ser reversible o al menos no impedir medidas posteriores. Por ello, aplicaremos estos criterios:

- Reversible, cuando el elemento queda exactamente igual si se elimina la medida. (Valoración: 3 ).
- No totalmente reversible, cuando el elemento se ve afectado si se elimina la medida, pero sigue conservando los valores por los que está protegido. (Valoración: 2).

- Irreversible, si el elemento se ve afectado de manera importante o no es posible una intervención posterior sin afectar sustancialmente al elemento. (Valoración: 1).

5.2.2. Indicadores relacionados con la rehabilitación de los sistemas constructivos (REH).

a) VIABILIDAD TÉCNICA (REH_01_vt)

Este indicador evalúa en qué grado es viable la solución desde el punto de vista técnico teniendo en cuentas los recursos disponibles. Los criterios de puntuación serían los siguientes:

- Viabilidad alta, cuando se trata de un producto comercial y accesible en el mercado. (Valoración: 3).

- Viabilidad media, si sólo se comercializa en puntos de distribución concretos y existe disponibilidad del material entre 15 y 30 días. (Valoración: 2).

- Viabilidad baja, cuando se trata de un producto experimental o de exportación y su plazo de entrega es superior a 30 días. (Valoración: 1).

b) VIABILIDAD ECONÓMICA (REH_O2_ve)

En este indicador evalúa de forma conjunta dos aspectos importantes. Por un lado, si la solución puede ser asumible desde el punto de vista económico y además si es una solución con un precio de mercado por encima o por debajo de las soluciones similares que suelen utilizarse para garantizar la misma prestación.

- Viabilidad alta, cuando su coste se adapta al presupuesto o es la única solución existente de su clase, es decir, no existe solución parecida en el mercado. (Valoración: 3).

- Viabilidad media, si tiene un precio con un margen de un $5 \%$ por encima que las soluciones parecidas para el mismo problema. (Valoración: 2).

- Viabilidad baja, cuando no entra dentro del presupuesto o tiene un precio con un margen de más de $5 \%$ por encima que las soluciones parecidas para el mismo problema. (Valoración: 1).

Para este indicador, como se trata de evaluar soluciones de rehabilitación energética, es necesario interrelacionar los criterios de la viabilidad económica con los de la mejora de la eficiencia energética. Por esta razón, el sistema ha incluido un índice comparativo de la viabilidad económica (Icve): que indica el porcentaje de ahorro energético por cada euro invertido, con el objetivo de poder comparar las soluciones:

$$
\text { Icve }=\frac{\operatorname{PRP}(\%)}{\text { Coste }_{\mathrm{s}}}
$$

Icve $=$ Índice comparativo de viabilidad económica. $\mathrm{PRP}(\%)=$ Porcentaje de reducción de pérdidas de calor. Coste $_{\mathrm{s}}=$ Coste en euros de la solución.

Para que la comparación con otras soluciones sea útil será importante aplicar las mismas condiciones en los casos a comparar, si se ha aplicado el IVA, costes indirectos, p.p. de medios auxiliares, etc. 
c) FACILIDAD DE EJECUCIÓN (REH_o3_fe)

Con este indicador se evalúa la facilidad de ejecución de la solución a adoptar. En este caso, se recogen los criterios orientativos para elegir cada una de las opciones. A modo orientativo proponemos:

- Viabilidad alta, cuando su instalación es sencilla, precisa pocos recursos (humanos y de medios auxiliares) y no presenta riesgos graves en su proceso. (Valoración: 3).

- Viabilidad media, si presenta una complejidad media para su instalación y recursos por encima de lo normal, aunque no existan riesgos graves en su instalación. (Valoración: 2)

- Viabilidad baja, cuando solo puede ser instalada por profesionales especializados y precisa supervisión y/o presenta dificultades en su manipulación, medios auxiliares especiales o riesgos graves en su manipulación o instalación. (Valoración: 1).

\section{d) FACILIDAD DE MANTENIMIENTO (reh o4 fm)}

Con este indicador se evalúa la dificultad o coste del mantenimiento de la solución. Se puede emplear como criterio la facilidad de la ejecución del mantenimiento, la periodicidad o el coste del mismo. A modo de orientación podría ser:

- Alta, si está en zona fácilmente accesible, precisa un periodo de mantenimiento superior al anual y/o su coste no supera el 10\% del coste del producto. (Valoración: 3).

- Media, si existe cierta dificultad para acceder a la zona donde se encuentra, si precisa un mantenimiento anual y/o su coste se encuentra entre el 10 y el 30\% del coste del producto. (Valoración: 2).

- Baja, si está en zona de difícil acceso, precisa un mantenimiento de varias veces al año y/o su coste supera el 30\% del coste de la medida. (Valoración: 1 ).

5.2.3. Indicadores relacionados con la rehabilitación energética (ENE).

a) MEJORA EFICIENCIA ENERGÉTICA (ENE_o1_me) El objeto del sistema es calificar o evaluar el edificio completo que se quiere rehabilitar energéticamente; se trata de establecer un sistema de cribado de soluciones como paso previo a que el técnico que realice la calificación energética pueda incluir ya soluciones que previamente se ha comprobado que cumplen con los requisitos de la catalogación. No obstante, es importante incluir indicadores que nos ayuden a elegir soluciones que teóricamente mejoren la eficiencia energética. Teniendo en cuenta que el sistema está diseñado para evaluar soluciones de rehabilitación energética relacionadas con la envolvente, se han seguido los mismos parámetros característicos de la envolvente térmica recogidos en el CTE (19), el valor de la transmitancia térmica del elemento y la permeabilidad al aire en huecos.

El CTE utiliza el valor de la transmitancia térmica del elemento como valor orientativo, por lo que nos valdremos del mismo dato para configurar el indicador, con la salvedad de que lo que haremos será calcular el tanto por ciento de mejora teniendo en cuenta las pérdidas de calor a través del elemento. Si tuviéramos sólo en cuenta la transmitancia del elemento, sin tener en cuenta la superficie en $\mathrm{m}^{2}$ en los que se aplica la solución, al comparar soluciones para un mismo elemento, podríamos encontrar que se penalizarían soluciones con una transmitancia más alta pero que se podría aplicar a toda la superficie. Por ello, este indicador presenta dos posibilidades. En primer lugar, si con la aplicación de la solución la transmitancia del elemento rehabilitado $\left(U_{\text {rev }}\right)$ alcanzara los límites de establecidos en el CTE, podríamos considerarla con el valor más alto posible. La otra opción es que se evalúe en función del porcentaje de reducción de pérdidas de calor como ya se ha venido haciendo en otros documentos como la Guía renovar para consumir menos energía (20), que consistiría en:

$$
\operatorname{PRP}(\%)=\frac{\left[(\mathrm{U} \cdot \mathrm{A})-\left(\mathrm{U}_{\text {rev }} \cdot \mathrm{A}_{\text {rev }}\right)\right] \cdot 100}{(\mathrm{U} \cdot \mathrm{A})}
$$

$\operatorname{PRP}(\%)=$ Porcentaje de reducción de pérdidas de calor. $\mathrm{U}=$ Transmitancia inicial del elemento $\left(\mathrm{W} / \mathrm{m}^{2} \mathrm{~K}\right)$.

$\mathrm{A}=$ Área del elemento en metros cuadrados.

$\mathrm{U}_{\text {rev }}=$ Transmitancia total del elemento rehabilitado $\left(\mathrm{W} / \mathrm{m}^{2} \mathrm{~K}\right)$.

$\mathrm{A}_{\text {rev }}=$ Superficie rehabilitada en metros cuadrados.

En este sentido, se han estudiado los elementos constructivos de los monasterios con respecto al cumplimiento de los valores orientativos recogidos en el CTE (21), obteniendo los valores iniciales de la transmitancia en muros desde 0,90 a $2,5 \mathrm{~W} / \mathrm{m}^{2} \mathrm{~K}$, en cubiertas inclinadas 2,08$2,78 \mathrm{~W} / \mathrm{m}^{2} \mathrm{~K}$, en suelos entre $3,5-3,98 \mathrm{~W} / \mathrm{m}^{2} \mathrm{Ky}$ en ventanas entre $4,3-5,75 \mathrm{~W} / \mathrm{m}^{2} \mathrm{~K}$. Por esta razón, aun siendo datos orientativos, hemos considerado que un valor de más de un $40 \%$ de porcentaje de reducción de pérdidas en muros y cubiertas, un 30\% en ventanas y un 50\% en suelos o un $\mathrm{U}_{\text {rev }}$ con un valor dentro de los límites establecidos por el CTE, puede valorarse como una mejora de la eficiencia alta (Valoración: 3). Un porcentaje entre $10 \mathrm{y}$ 40 en muros y cubiertas, de un 10 a un $30 \%$ en ventanas y de un 10 a un $50 \%$ en suelos podría considerarse como una mejora media (Valoración: 2). Cuando el porcentaje de reducción de pérdidas de energía (PRP) sea menos de un 10\% lo consideraríamos baja (Valoración: 1).

b) MEJORA DE LA PERMEABILIDAD AL AIRE DE HUECOS (ENE_01_pa)

Este es un indicador adicional que se ha creado específicamente para el caso de que el elemento a rehabilitar sea un hueco. La correlación corresponde con los valores ya indicados por el CTE HE (19) teniendo en cuenta las zonas climáticas según la rigurosidad del invierno, Tabla 2.

c) RESPETUOSA CON EL MEDIO AMBIENTE:

Este indicador evalúa las emisiones de $\mathrm{CO}_{2 \text { EQUIVALENTE }}$ que han sido necesarias para fabricar el material. El criterio elegido para su valoración es el "valor del coste energético de producción $\mathrm{MJ} / \mathrm{kg}$ ". Para calcular los rangos orientativos, hemos utilizado los siguientes datos: Los aportados por el Catálogo de soluciones constructivas de rehabilitación (7) para aislantes, los recogidos en la tabla 1 del artículo Modelo de cuantificación del consumo energético en edificación (22), la base de datos ICE (23) y los datos recogidos por Rúa y López Mesa (24). Como existe gran disparidad de datos, hemos utilizado consensuado todos ellos llegando a los criterios siguientes: 
Tabla 2. Criterios de elección asignados al indicador Permeabilidad al Aire de Huecos.

\begin{tabular}{|c|c|c|c|c|c|}
\hline \multirow{2}{*}{$\begin{array}{c}\text { Permeabilidad al aire } \\
\text { de huecos }\end{array}$} & \multicolumn{4}{|c|}{ Criterio } & \multirow{2}{*}{ Correspondencia numérica } \\
\hline & $\mathbf{A}$ & $\mathbf{B}$ & $\mathbf{D}$ & $\mathbf{E}$ & \\
\hline Muy estanco & \multicolumn{2}{|c|}{$\mathrm{X} \leq 50 \mathrm{~m}^{3} / \mathrm{hm}^{2}$} & \multicolumn{2}{|c|}{$\mathrm{X} \leq 27 \mathrm{~m}^{3} / \mathrm{hm}^{2}$} & 3 \\
\hline Estanco & \multicolumn{2}{|c|}{$50<\mathrm{X} \leq 100 \mathrm{~m}^{3} / \mathrm{hm}^{2}$} & \multicolumn{2}{|c|}{$27<\mathrm{X} \leq 50 \mathrm{~m}^{3} / \mathrm{hm}^{2}$} & 2 \\
\hline Poco estanco & \multicolumn{2}{|c|}{$\mathrm{X}>100 \mathrm{~m}^{3} / \mathrm{hm}^{2}$} & \multicolumn{2}{|c|}{$\mathrm{X}>50 \mathrm{~m}^{3} / \mathrm{hm}^{2}$} & 1 \\
\hline
\end{tabular}

- Alta, cuando el valor del coste energético de producción sea menor de $25 \mathrm{MJ} / \mathrm{W}$. (Valoración: 3).

- Media, si el valor del coste energético de producción está entre 25 y $50 \mathrm{MJ} / \mathrm{W}$. (Valoración: 2).

- Baja, si el valor del coste energético de producción es mayor de $50 \mathrm{MJ} / \mathrm{W}$. (Valoración: 1).

d) DURABILIDAD (ENE_03_du)

Aquí se evalúa la durabilidad de la medida; es decir, cuál será su periodo de vida o cuánto tardaremos en remplazarla. Es un dato a tener en cuenta, dado que proporciona información útil para posteriormente poder calcular el plazo de amortización de la medida. Además, desde el punto de vista energético, un material con una durabilidad baja, supondrá asumir un nuevo coste energético de producción con cada reposición durante la vida útil del edificio. Según el criterio establecido los materiales con una alta durabilidad serán aquellos que puedan durar más de 25 años (Valoración: 3), los de baja durabilidad los que no lleguen a los 10 años (Valoración: 1) y si está entre 10 y 25, (Valoración: 2).

\section{SISTEMA DE EVALUACIÓN}

Conocidos los indicadores, el proceso a seguir en la evaluación es el siguiente:

a) Se introducen en la herramienta los datos del elemento constructivo sobre el que se va a intervenir.

b) Posteriormente debe seleccionarse una de las cinco categorías o CASOS de catalogación establecidas para el elemento constructivo.

c) Determinar la zona climática según CTE.

d) Determinar el espesor y superficie del elemento a intervenir.

e) Deben incorporarse los datos de la solución a evaluar, que son la base para establecer las valoraciones de los indicadores:

- Descripción.

- Color del acabado.

- Textura del acabado.

- Espesor de la solución (m).

- Si afecta o modifica la apariencia del elemento constructivo actual.

- Composición química o tipo de material.

- Modo de anclaje o fijación al elemento constructivo.

- Si es un producto comercial, accesible, plazos de entrega, medios auxiliares, etc.

- Coste económico de la solución (euros).

- Dificultades técnicas para su ejecución.

- Ventajas y problemas sobre su mantenimiento.

- Coste energético de la solución (MJ/W).
- Durabilidad en años.

- Superficie del elemento constructivo en la que se aplica la solución (m2).

También es necesario aportar otros datos como:

- En caso de tratase de un hueco, permeabilidad al aire.

- Transmitancia total elemento rehabilitado (Urev).

- Porcentaje de reducción de pérdidas de energía (PRP).

- Índice comparativo de viabilidad económica (Icve).

Una vez introducidos los datos, se valoran en primer término los indicadores correspondientes a la catalogación del edificio (CAT). Estos indicadores tienen la función de aceptar o descartar la solución y establecen valores obligatorios según las diferentes categorías analizadas en el apartado 5.1. Si la solución aparece como aceptada, el sistema procede a valorar el resto de los indicadores.

Finalmente, como hemos indicado, el sistema ofrece una valoración parcial por cada indicador y la valoración total de la solución. Además, se obtiene una representación gráfica de la valoración. Por un lado, los Indicadores de la categoría CAT se representan mediante un gráfico radial y por otro los indicadores REH y ENE en un gráfico de columnas, que ofrecen información complementaria de la solución para poder establecer una comparativa entre diversas alternativas.

En el caso de los indicadores CAT, al disponer de un indicador en cada vértice de la gráfica, se aprecia la situación global de la solución, permitiendo superponer los patrones de las distintas soluciones para comprobar la más adecuada. En la Figura 1 arriba, se han representado las cinco categorías propuestas que determinan patrones que cumplen los requisitos mínimos de la catalogación, de modo que, cuanto más se acerque la solución evaluada a su patrón correspondiente, más posibilidades de aceptación tendrá.

Los patrones que deben cumplir los indicadores CAT y que vienen representados en la figura anterior, han sido obtenidos siguiendo la Tabla 3. Para cada CASO hemos establecido cual tendría que ser la puntuación para que cumpliera con los requisitos establecidos en la catalogación. Estas puntuaciones son las que hemos extrapolado a los vértices de los diagramas radiales. Sombreado en naranja, Tabla 3, vemos los valores obligatorios. Sombreado en gris, los requisitos establecidos para una rehabilitación (que se diferencie de los originales). No podríamos utilizar la valoración 3 que sería idéntico, pero el sistema permite que si con alguno de estos tres indicadores existe la posibilidad de no mimetización uno de ellos o dos de ellos puedan valorarse con la máxima puntuación. El sombreado azul hace mención al CASO4_NO_V, elemento no original integrado en una zona protegida, por lo 

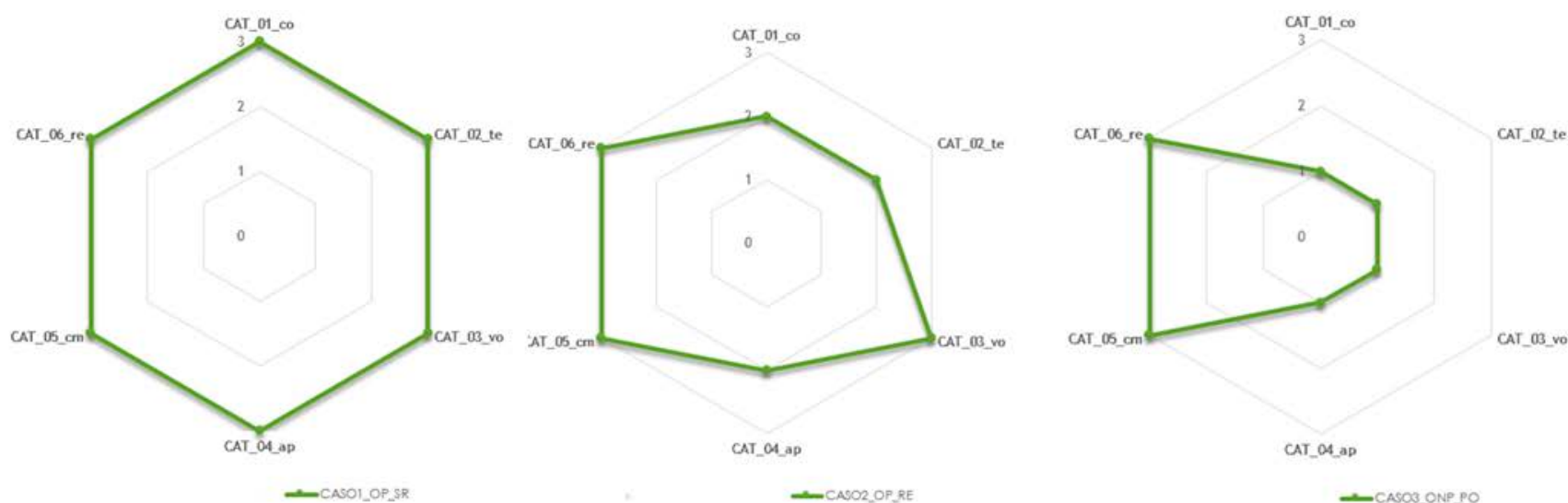

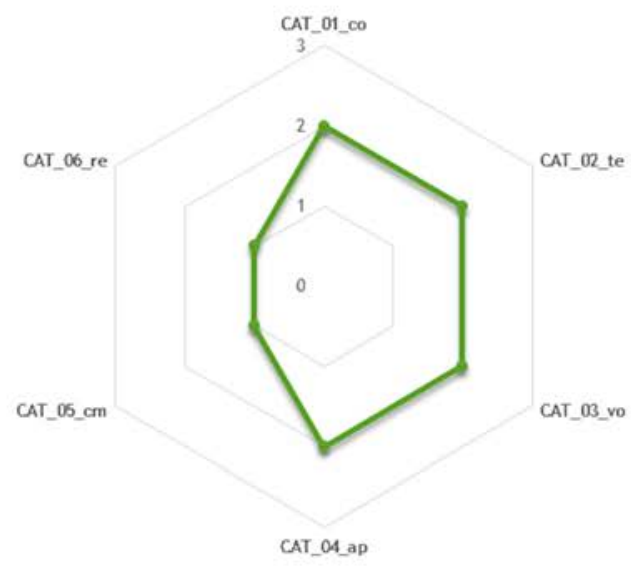

- CASOANO_V

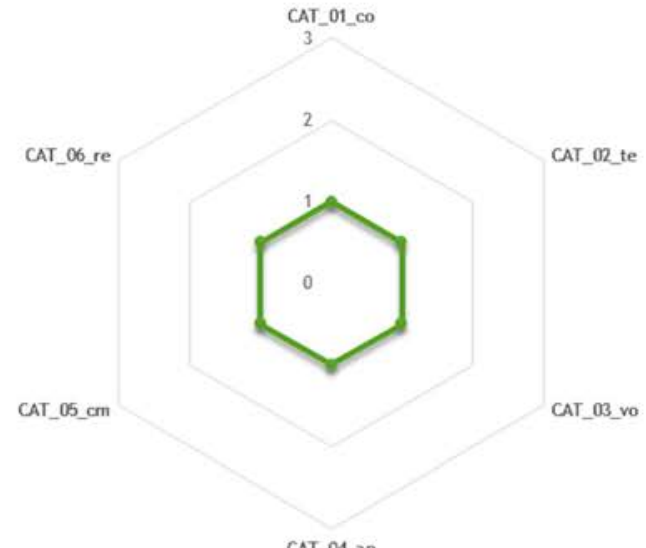

CAT_04_ap
Indicadores CAT

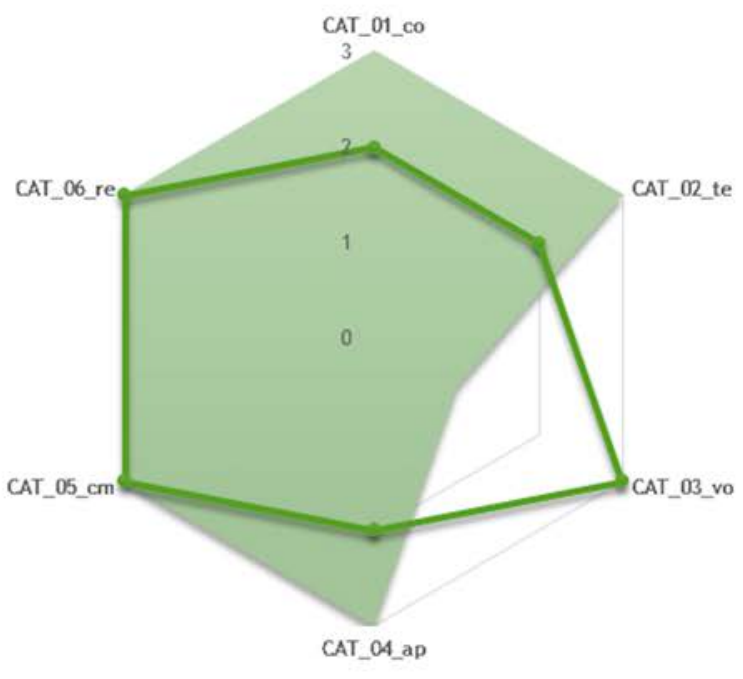

LCASO2_OP_RE
Indicadores CAT

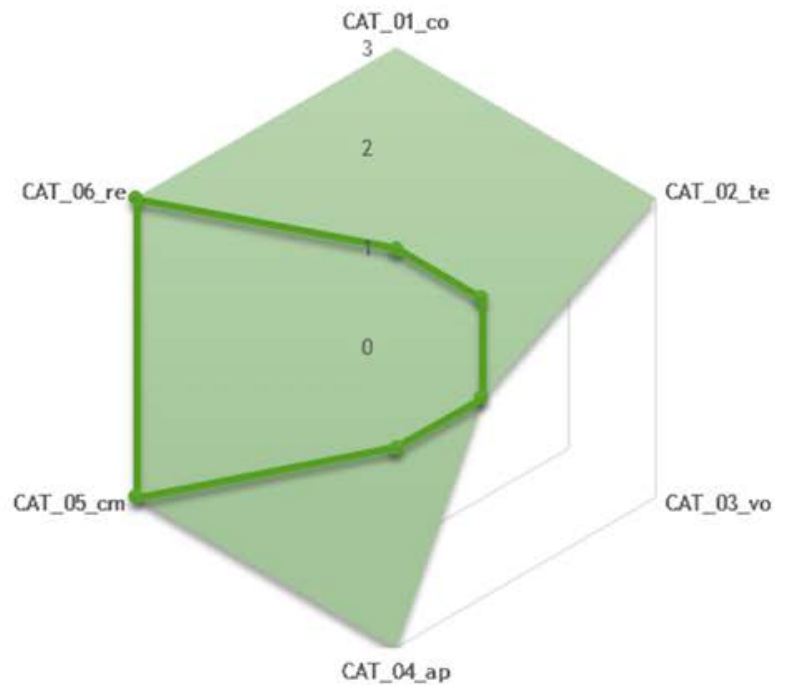

-CASO3_ONPPO

Figura 1. Arriba: representación de los diferentes patrones según los 5 CASOS. Abajo: comparativa de representación gráfica de una misma solución con dos patrones diferentes para los indicadores CAT. 
Tabla 3. Asignación de valores obligatorios y opcionales según el CASO y el indicador para aceptar o no una solución.

\begin{tabular}{|c|c|c|c|c|c|}
\hline & CASO1_OP_SR & CASO2_OP_RE & CASO3_ONP_PO & CASO4_NO_V & CASO5_NO_O \\
\hline \multicolumn{6}{|l|}{ CAT } \\
\hline CAT_01_co & 3 & $2(3)$ & $1-3$ & $2-3$ & $1-3$ \\
\hline CAT_02_te & 3 & 2(3) & $1-3$ & $2-3$ & $1-3$ \\
\hline CAT_03_vo & 3 & 3 & $1-3$ & $2-3$ & $1-3$ \\
\hline CAT_04_ap & 3 & 2(3) & $1-3$ & $2-3$ & $1-3$ \\
\hline CAT_05_cm & 3 & 3 & 3 & $1-3$ & $1-3$ \\
\hline CAT_o6_re & 3 & 3 & 3 & $1-3$ & $1-3$ \\
\hline \multicolumn{6}{|l|}{ REH } \\
\hline REH_01_vt & $1-3$ & $1-3$ & $1-3$ & $1-3$ & $1-3$ \\
\hline REH_02_ve & $1-3$ & $1-3$ & $1-3$ & $1-3$ & $1-3$ \\
\hline REH_O3_fe & 1-3 & $1-3$ & $1-3$ & $1-3$ & $1-3$ \\
\hline REH_O4_fm & $1-3$ & $1-3$ & $1-3$ & $1-3$ & $1-3$ \\
\hline \multicolumn{6}{|l|}{ ENE } \\
\hline ENE_01_me & $1-3$ & $1-3$ & $1-3$ & $1-3$ & $1-3$ \\
\hline ENE_01_pa & 1-3 & $1-3$ & $1-3$ & $1-3$ & $1-3$ \\
\hline ENE_02_rm & $1-3$ & $1-3$ & $1-3$ & $1-3$ & $1-3$ \\
\hline ENE_O3_du & $1-3$ & $1-3$ & $1-3$ & $1-3$ & $1-3$ \\
\hline
\end{tabular}

que para garantizar la armonía con el conjunto tendría que valorarse con más de 1.

En la Figura 1 abajo, hemos representado la misma solución evaluada (superficie sombreada) en relación con dos patrones de catalogación diferentes. La superficie que se encuentra dentro del patrón cumple todos los requisitos, mientras que la superficie que sale fuera del patrón es la que rebaja la puntuación. En la figura de la izquierda aparece el CASO2_OP_ RE y a la derecha CASO3_CNP_PO. En el caso de la izquierda la solución no estaría aceptada, ya que el perímetro del patrón sobresale del área sombreada de la solución, debido al volumen. En el gráfico de la derecha, la solución sería aceptada, pues el área sombreada queda dentro de los límites del patrón; es decir, se cumplirían las exigencias mínimas.

Para representar el resto de los indicadores se ha elegido un diagrama de columnas siguiendo el criterio de puntuación con un valor máximo de 3 y de 1 para el mínimo. Mientras más alta sea la columna mejor será el indicador parcial y en consecuencia la solución a evaluar.

\section{PERFILADO Y VALIDACIÓN DEL SISTEMA}

Para perfilar el sistema de evaluación y comprobar que evalúa correctamente las soluciones de rehabilitación energética, se realizó un banco de pruebas en el que se chequearon cinco soluciones de rehabilitación energética en tres monasterios BIC. Para ello nos valdremos de casos-tipo (25). Con esta opción buscamos que en la muestra aparezcan todas las variables que intervienen en el sistema de evaluación abogando menos por la cantidad de casos o elementos de muestreo y más por la representatividad de los mismos.

Las soluciones propuestas fueron:

- Colocación en muros de fachada de aislamiento por el interior, con trasdosado auto-portante;

- Cambio de ventanas existentes por otras más aislantes;
- Colocación de suelos térmicos sobre los existentes;

- Colocación de material aislante bajo cubierta, por el interior;

- Colocación de aislamiento por el exterior de la cubierta.

En concreto se han evaluado las siguientes soluciones para perfilar el sistema:

- En Santa María de Retuerta (Sardón de Duero, Valladolid):

- Colocación en muros de fachada de aislamiento por el interior.

- Huecos del claustro.

- Enlosado con baldosa cerámica de las pandas del claustro.

- Aislamiento térmico bajo la cubierta y por el exterior.

- En Santa María de la Vid (La Vid y Barrios, Burgos):

- Rehabilitación de un muro de la iglesia.

- Huecos del claustro.

- Enlosado de piedra en las pandas del claustro.

- Aislamiento térmico por el exterior en zona del Claustro y por el interior.

- En Santa María la Real (Aguilar de Campoo, Palencia):

- Rehabilitación de muro del refectorio.

- Huecos del claustro.

- Enlosado de la iglesia.

- Cubierta de la iglesia por el interior y por el interior.

En la Tabla 4 podemos ver la matriz predictiva que se diseñó en la que se establecían en verde las evaluaciones que debían salir favorables y en rosa aquellas que el sistema debía descartar.

Las conclusiones de esta aplicación para validar el sistema, se concretan en: 
Tabla 4. Representación de las predicciones en cuanto a aceptación o rechazo de las soluciones a evaluar.

\begin{tabular}{|c|c|c|c|c|}
\hline & Monasterio Retuerta & Monasterio La Vid & Monasterio La Real & \\
\hline Muros & $\begin{array}{l}\text { CASO1_OP_SR } \\
\text { (exterior) } \\
\text { CASO3_ONP_PO } \\
\text { (interior) }\end{array}$ & CASO1_OP_SR & CASO1_OP_SR & Solución 1 \\
\hline Huecos & CASO4_NO_V & CASO4_NO_V & CASO4_NO_V & Solución 2 \\
\hline Suelos & CASO5_NO_O & CASO3_ONP_PO & CASO5_NO_O & Solución 3 \\
\hline \multirow{2}{*}{ Cubierta } & $\begin{array}{l}\mathrm{CASO} 2 \text { _OP_RE } \\
\text { (exterior) } \\
\text { CASO3_ONP_PO } \\
\text { (interior) }\end{array}$ & $\begin{array}{c}\text { CASO3_ONP_PO } \\
\text { (interior) } \\
\text { CASO4_NO_V } \\
\text { (exterior) }\end{array}$ & $\begin{array}{l}\text { CASO4_NO_V } \\
\text { (exterior) } \\
\text { CASO5_NO_O } \\
\text { (interior) }\end{array}$ & Solución 4 \\
\hline & $\begin{array}{l}\mathrm{CASO} 2 \_\mathrm{OP} \_\mathrm{RE} \\
\text { (exterior) } \\
\text { CASO3_ONP_PO } \\
\text { (interior) }\end{array}$ & $\begin{array}{l}\text { CASO3_ONP_PO } \\
\text { (interior) } \\
\text { CASO4_NO_V } \\
\text { (exterior) }\end{array}$ & $\begin{array}{l}\text { CASO4_NO_V } \\
\text { (exterior) } \\
\text { CASO5_NO_O } \\
\text { (interior) }\end{array}$ & Solución 5 \\
\hline
\end{tabular}

Verde: aceptado / Rosa: rechazado.

a) En todos los casos, ha sido posible aplicar el sistema para realizar la evaluación.

b) Hemos podido confirmar que las soluciones cumplen, en todos los casos, con la catalogación correspondiente. De hecho, algunas son soluciones reales que se han llevado a la práctica.

c) Hemos podido comprobar cómo la misma solución resulta con diferente valoración en función de las características del edificio.

d) Se confirma que el sistema permite comparar las soluciones desde un punto de vista económico y energético.

Una vez calibrado y puesto en marcha el sistema, decidimos validarlo con un caso externo. En este caso se emplea la intervención en los patios del Colegio Alfonso XII del Monasterio de El Escorial (26):

- Restauración de las armaduras de pares, el enripiado de madera y la cobertura de los faldones de cubierta de pizarra, manteniendo la configuración original (CASO2_OP_ $\mathrm{RE}$;

- Cambio de la carpintería de madera en ventanas de buhardillas, incorporando vidrio doble de 6+4+4 mm (CASO4 NO_V);

Ambas soluciones no podían ser descartadas por el sistema de evaluación, ya que habían sido aceptadas y aprobadas en el caso real. Una vez realizada la evaluación, las dos soluciones analizadas con SESREBIC (16) fueron aceptadas como indicamos en la Figura 2.

\section{CONCLUSIONES}

La conclusión principal es que se ha conseguido diseñar un sistema que permite valorar las distintas soluciones de rehabilitación energética para aplicarlo a edificaciones declaradas BIC, que no se fundamenta exclusivamente en aspectos económicos y energéticos, sino que también incluye los indicadores necesarios para respetar protección patrimonial del edificio.

En esta primera fase, el sistema se ha enfocado en la tipología constructiva del monasterio, pero dado el resultado positivo de su aplicación, se abre la puerta a la continuación de la investigación en otras tipologías edificatorias.

El sistema de valoración, se ha implementado en una herramienta simplificada que hemos denominado SESREBIC (16), que incorpora indicadores CAT relacionados con los criterios más significativos utilizados por las Comisiones de patrimonio encargadas de otorgar la calificación BIC; indicadores relacionados con la eficiencia energética que aporta la solución ENE e indicadores relacionados con las características constructivas de la solución propuesta REH.

Para la validación de la herramienta, se ha aplicado en un monasterio declarado BIC, y que ha sido sometido a una intervención de rehabilitación energética, comprobando la excelente respuesta de la herramienta de evaluación.

En cuanto a las conclusiones sobre el sistema SESREBIC, destacamos:

a) Se trata de una herramienta que permite al proyectista el comprobar si la solución elegida, cumplirá con los requisitos establecidos en la protección del mismo, de forma que sirva tanto para aceptar y comparar como para descartar soluciones, ya que si una solución que parece adecuada "a priori", no respeta los criterios de catalogación del edificio, no tendrá sentido incluirla en los programas de simulación energética.

b) Es de fácil aplicación, ya que se ha diseñado a partir de una hoja de cálculo, en la que, de una manera sencilla y sistemática, se incorporan una serie parámetros específicos relacionados con los tres tipos de indicadores.

c) La herramienta también es de fácil actualización, ya que los rangos establecidos en los criterios pueden modificarse fácilmente si hiciera falta y además podrían incluirse más indicadores llegado el caso.

d) El sistema permite comparar las soluciones por diferentes métodos: Por comparativa de puntuaciones totales, por comparativa de índices generales y por comparativa de índices específicos como el porcentaje de pérdidas y el índice comparativo de viabilidad económica. Además, es posible comparar soluciones en diferentes elementos y como diferentes categorías de catalogación. 


\begin{tabular}{|c|c|c|c|c|}
\hline INDICADORES & Código & Valoración & Puntuación & Cumplimiento requisilos calalogación \\
\hline Color & CAT_Ol_co & Parecido & 2 & cumple \\
\hline Textura & CAT_02_te & Idéntico & 3 & cumple \\
\hline Volumen & CAT_03_vo & Idéntico & 3 & cumple \\
\hline Apariencia & CAT_04_ap & Parecido & 2 & cumple \\
\hline Compatibilidad & CAT_05_cm & Compatible & 3 & cumple \\
\hline Reversibilidad & CAT_06_re & Reversible & 3 & cumple \\
\hline & & & & solución acepłac \\
\hline Viabilidad técnica & REH_Ol_vt & Media & 2 & \\
\hline Viabilidad económica & REH_O2_ve & Media & 2 & \\
\hline Facilidad de ejecución & REH_O3_fe & Alta & 3 & \\
\hline Facilidad de mantenimiento & REH_O4_fm & Alta & 3 & \\
\hline Mejora de la eficiencia energética & ENE_01_me & Media & 2 & \\
\hline Mejora de la permeabilidad al aire & ENE_01_pa & Poco_estanco & 1 & \\
\hline Respetuosa con el Medio Ambiente & ENE_02_rm & Sin_datos & 0 & \\
\hline Durabilidad & ENE_03_du & Alta & 3 & \\
\hline
\end{tabular}

\section{Indicadores CAT}
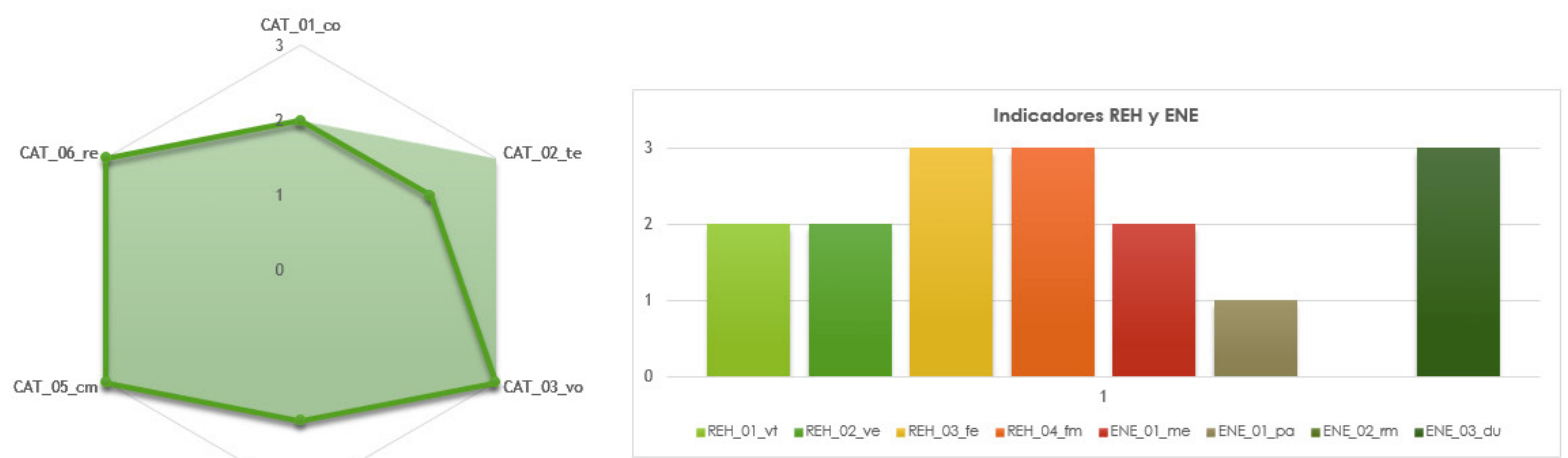

CAT_04_ap

Solución evaluada 1 -CASO2_OP_RE

\begin{tabular}{|l|l|l|l|}
\hline CASO & CASO2_OP_RE & Elemento & CUBIERTA \\
\hline
\end{tabular}

\begin{tabular}{|c|c|c|c|c|}
\hline INDICADORES & Código & Valoración & Puntuación & Cumplimiento requisitos catalogación \\
\hline Color & CAT_01_CO & Parecido & 2 & cumple \\
\hline Textura & CAT_02_te & Idéntico & 3 & cumple \\
\hline Volumen & CAT_03_vo & Idéntico & 3 & cumple \\
\hline Apariencia & CAT_04_ap & Parecido & 2 & cumple \\
\hline Compatibilidad & CAT_05_cm & Compatible & 3 & cumple \\
\hline Reversibilidad & CAT_06_re & Reversible & 3 & cumple \\
\hline & & & & solución aceptac \\
\hline Viabililad técnica & REH_01_vt & Media & 2 & \\
\hline Viabilílad económica & REH_O2_ve & Media & 2 & \\
\hline Facilidad de ejecución & REH_03_fe & Media & 2 & \\
\hline Facilidad de mantenimiento & REH_04_fm & Media & 2 & \\
\hline Mejora de la eficiencia energética & ENE_01_me & Media & 2 & \\
\hline Mejora de la permeabilidad al are & ENE_01_Pa & No_aplica & 0 & Seleccionar No aplica \\
\hline Respetuosa con el Medio Ambiente & ENE_O2_tm & Sin_datos & 0 & \\
\hline Durabilidad & ENE_03_du & Media & $7 \quad 2$ & \\
\hline
\end{tabular}

Indicadores CAT
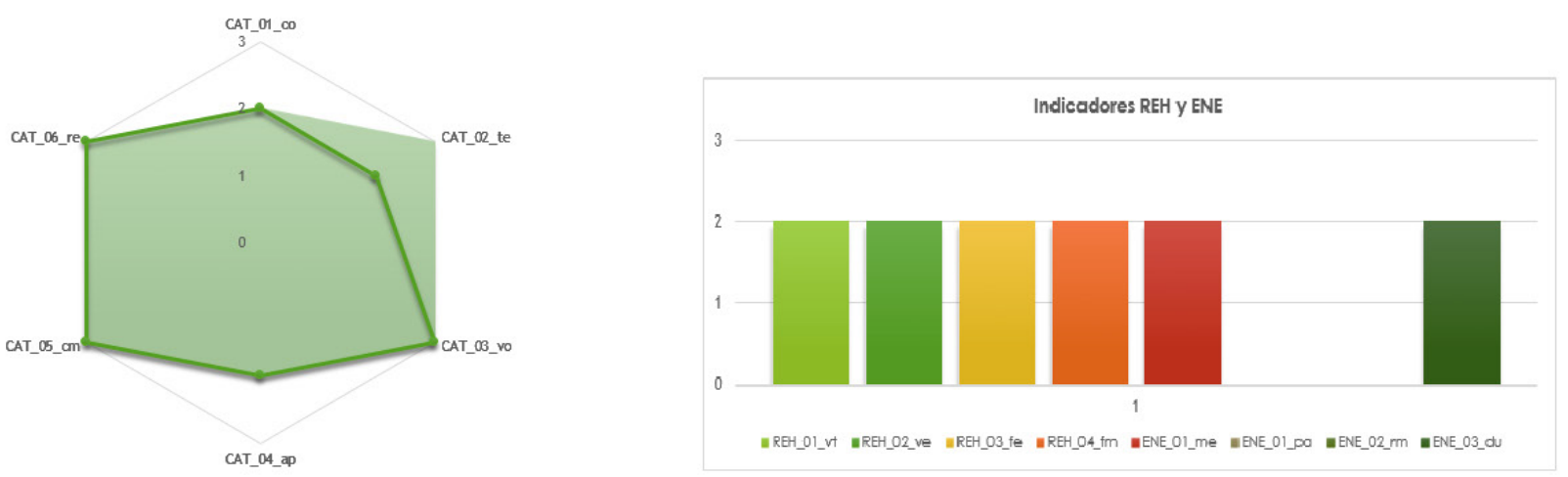

- Solución evoluada -CASO2_OP_RE

Figura 2. Evaluaciones soluciones Monasterio del Escorial con SESREBIC. 
Por último, señalar que el presente trabajo proporciona una primera respuesta al RD 564/2017. Este nuevo RD, modifica el artículo 2.2.a del anterior, señalando que se excluyen de la certificación de la eficiencia energética "los edificios protegidos oficialmente por ser parte de un entorno declarado o en razón de su particular valor arquitectónico o histórico, siempre que cualquier actuación de mejora de la eficiencia energética alterase de manera inaceptable su carácter o aspecto, siendo la autoridad que dicta la protección oficial quien determine los elementos inalterables”. En este sentido, para poder cumplir con este precepto, será necesario o bien realizar el certificado energético aportando en las medidas de mejora aquellas soluciones que no alteren estos valores patrimoniales; o bien, justificar mediante alguna herramienta o procedimiento, que cualquier medida de mejora afectaría a la catalogación del edificio para no realizar el certificado. De una forma u otra la existencia de herramientas que ayuden al cumplimiento de la norma serán esenciales.

\section{REFERENCIAS}

(1) Ministerio de la Presidencia (2013). Real Decreto 235/2013, de 5 de abril, por el que se aprueba el procedimiento básico para la certificación de la eficiencia energética de los edificios. Boletín Oficial del Estado, nº89. España.

(2) Ministerio de Educación, Cultura y Deporte (2010). Consulta Online a la Base de datos de bienes inmuebles. http:// www.mecd.gob.es/bienes/cargarFiltroBienesInmuebles.do?layout=bienesInmuebles\&cache=init\&language=es. [Acceso: 29-06-2018].

(3) Ministerio de la Presidencia y para las administraciones territoriales (2017). Real Decreto 564/2017, de 2 de junio, por el que se modifica el Real Decreto 235/2013, de 5 de abril, por el que se aprueba el procedimiento básico para la certificación de la eficiencia energética de los edificios. Boletín Oficial del Estado, $n^{0} 134$. España.

(4) Jefatura del Estado (1985). Ley 13/1985, de 25 de junio, Ley del Patrimonio Histórico Español. Boletín Oficial del Estado, $\mathrm{n}^{\mathrm{0}} 155$. España.

(5) Ramos Vilariño, E. (2012, 3 de octubre). Unidades de vidrio aislante de aislamiento térmico reforzado. Guía de Materiales aislantes y eficiencia energética. Madrid (FENERCOM).

(6) De Isabel, J., García, M., Egido, C. (2009). Guía de auditorías energéticas en el sector hotelero de la Comunidad de Madrid. Madrid. Dirección General de Industria, Energía y Minas de la Consejería de Economía y Hacienda y la Fundación de la Energía de la Comunidad de Madrid.

(7) Serrano-Lanzarote, B., García-Prieto Ruiz, A., Ortega Madrigal., L. (2011). Catálogo de soluciones constructivas de rehabilitación, p. 321. Valencia: Generalitat Valenciana. Consellería de Medio Ambiente, Agua, Urbanismo y Vivienda.

(8) MIYABI y el Centro Nacional de Energías Renovables (CENER) (2012). Guía IDAE: Manual de usuario de calificación energética de edificios existentes $\mathrm{CE}_{3} \mathrm{X}$. Madrid: IDAE.

(9) Perán, J., Martín, P., Bujedo, L. (2014, 30 de septiembre). Renerpath: metodología de rehabilitación energética de edificios patrimoniales. En International Conference Energy Efficiency in historic buildings, (pp. 543-552). Madrid: Ars Civilis y Casas Históricas.

(10) Effesus (2012). Proyecto Effesus. http://www.effesus.eu

(11) EURAC research (2010). Proyecto 3encult. http://www.3encult.eu/en/project/welcome/default.html

(12) Garcia-Almirall, M. (2010, 6 de octubre) Prototipo SIG para la gestión patrimonial de suelo. En International Conference Virtual City and Territory, $6^{\circ}$ Congreso Internacional Ciudad y Territorio Virtual, Mexicali: UABC. http://hdl.handle. net/2099/12801

(13) HeritageCARE. http://heritagecare.eu/

(14) PetroBIM. http://petrobim.com/

(15) MHS - Monitoring Heritage System. http://www.mhsproject.com/es/inicio/

(16) López Zambrano, M.J. (2017). Sistema de evaluación de soluciones de rehabilitación energética en monasterios bien de interés cultural. (Tesis Doctoral Inédita). Sevilla: Universidad de Sevilla.

(17) IPCE (2011). Plan Nacional de Abadías, Monasterios y Conventos. Ministerio de Educación, Cultura y Deporte, Madrid, p. 134.

(18) ICOMOS (2004). Recomendaciones para el análisis, conservación y restauración estructural del patrimonio arquitectónico. International Scientific Committee for Analysis and Restoration of Structures of Architectural Heritage.

(19) CTE (2017). Documento Básico HE Ahorro de energía. Apartado 2.2.1.2., tabla 2.3. Ministerio de Fomento. España.

(20) Alba Ingenieros Consultores (2015). Renovar para consumir menos energía. Rehabilitación energética de edificios y viviendas. Madrid: Fenercom.

(21) CTE (2017). Documento Básico HE Ahorro de energía. Apéndice E. Apartado E.2. Parámetros característicos de la envolvente térmica. Tablas E.1. y E.2. Ministerio de Fomento. España.

(22) Mercader, M., Olivares, M., Ramírez de Arellano, A. (2012). Modelo de cuantificación del consumo energético en edificación. Materiales de Cosntrucción, 62(308): 567-582, doi: https://doi.org/10.3989/mc.2012.02411.

(23) Hammond, G., Jones, C. (2011). A BSRIA guide Embodied Carbon The Inventory of Carbon and Energy (ICE), p. 136. University of Bath.

(24) Ruá, J., López-Mesa, B. (2012). Certificación energética de edificios en España y sus implicaciones económicas. Informes de la Construcción, 64(527):307-318, doi: https://doi.org/10.3989/ic.11.028.

(25) Hernández Sampieri, R., Fernández Collado, C., Baptista Lucio, P. (2012). Metodología de la Investigación, $5^{\text {a }}$ Edición. México D.F.: Mc Graw Hill.

(26) Sancho, J. (2015). Restauración de cubiertas de los patios del colegio de Alfonso XII en el Monasterio de El Escorial. Memoria histórica. Madrid: Patrimonio Nacional. Dirección de Inmuebles y Medio Natural. 